\title{
We have arrived to the time when the bottom is starting to fill up!
}

\author{
Maria A. Croyle ${ }^{1,2}$
}

Published online: 13 December 2017

(c) The Korean Society of Pharmaceutical Sciences and Technology 2017

If one looks back through the literature, it will quickly become apparent that the concept of "nanotechnology" was first conceptualized through the genius, personality and eloquence of Richard Feynman in his landmark lecture "There's plenty of room at the bottom" given at a meeting of the American Physical Society at the California Institute of Technology in 1959 (Feynman 1960). It was clear that the forward-thinking ideas presented by Feynman ignited the collective imagination as the ability to manipulate individual atoms and molecules to form macroscopic objects was illustrated by "replicator machines" described in the science fiction series Star Trek (1966) and technology capable of allowing humans to analyze common material objects at the nano level shown in the film Fantastic Voyage (1966). With regard to the importance and potential impact that nanotechnology holds in global health and medicine, considerable funding was also allocated and invested in the development of this multi-disciplinary field in many countries such as the United States, Korea, Japan, Australia, the European Union and several individual member states for more than half a century. If this is true, why then, do we not have the Star Trek replicators in every home around the world in 2018 ? Why is each physician's room not equipped with machines that allow the doctor to shrink to microscopic size to treat illness? Clearly, progress toward making these things a reality has not been impeded by lack of ingenuity and support but, instead, by the inability of technology to keep up with the pace of our dreams.

This special issue brings together a rich variety of manuscripts that illustrate that technology is starting to catch up at an unprecedented rate and is significantly impacting how both new and established medicinal compounds are

Maria A. Croyle

macroyle@austin.utexas.edu

1 University of Texas at Austin College of Pharmacy, Austin, TX, USA

2 Division of Molecular Pharmaceutics and Drug Delivery, Austin, TX 78712, USA formulated and delivered today. This can first be seen in the manuscript by Hong and Choi in which mesoporous silicabased nanoparticles serve as the basis of "smart medicines", capable of delivering more than one medicinal agent to a specific area in the body in response to external cues. Technological advancement is also reflected in the manuscript by Heprin and Smyth which provides an excellent example of how a highly innovative method for production of fine, monodisperse drug particles significantly improves the physicochemical properties of poorly water soluble drugs.

The manuscript by Liu et al. provides an exceptional summary of recent and emerging technological breakthroughs which now allow us to evaluate both new and traditional dosage forms on the nano scale and summarizes how data garnered from these techniques have significantly improved methods for formulation selection and manufacturing processes for complex, poorly water soluble drugs. In line with this theme, the manuscript by Ghosh et al. describes a novel technology for high-throughput screening of peptides with properties suitable for enhancing drug delivery to a variety of difficult to reach targets such as the blood-brain barrier and the solid tumor microenvironment.

In his famous lecture, Feynman explains that the now ever famous phrase "There is plenty of room at the bottom" implies that as we start preparing and analyzing materials at the micro then nano level, space widens due to the small amount of it that we take up through miniaturization. As more nanomedicines begin to receive approval from regulatory agencies and enter the market, they will begin to fill this vast space. The review by Choi and Han provides a comprehensive review of nanomedicines currently on the market. The insightful discussion of issues that must be considered during the assessment of pharmacokinetic profiles of agents formulated in these novel dosage forms according to US FDA and EMA guidelines will be invaluable to anyone interested in developing nanomedicines suitable for clinical testing.

In the last half of 2017, two novel gene medicines [Kymriah $^{\mathrm{TM}}$ (Novartis) and Yescarta ${ }^{\mathrm{TM}}$ (NCI/Kite)] gained US FDA approval while another is slated for early 2018 
[Luxturna ${ }^{\mathrm{TM}}$ (Spark Therapeutics)], almost 3 decades after the first gene therapy treatments entered clinical trials. The manuscript by Jonsson-Schmunk et al. provides a review of the current understanding of how these and other nanomedicines might impact hepatic drug metabolism via the cytochrome $\mathrm{P} 4503 \mathrm{~A} 4$ pathway and highlights the fact that additional, long-term mechanistic studies are needed to fully understand how complex nanomedicines exert their effect on CYP and other biological systems that play key roles in drug metabolism and excretion.

The review by Ma and Williams provides a comprehensive analysis of how nanotechnology has improved oral drug absorption. Nguyen et al. expertly summarizes the characteristics of a variety of cell penetrating peptides developed to improve delivery of nanomedicines across the skin, intranasal and ocular mucosa. Both of these papers realistically, and laudably, highlight challenges that remain in the scale-up and manufacture of many nanoparticle-based delivery systems, which, at the moment, is most likely the rate limiting step for filling the vast space opened up by Feynman and the concept of working at the nanoscale. However, these provide valuable insight to those interested in developing and improving nanomedicines for future clinical use.

In closing, I would like to thank the authors for their contributions to advancing our current understanding of the field and realizing the work that lies ahead. Many thanks to the coordinating editor, Professor Dong Hee $\mathrm{Na}$, for his excellent assistance in compiling this volume and to Professor Hyo-Kyung Han for the encouragement to develop this special issue. I hope that you enjoy the collection of manuscripts that we have assembled and find them to be insightful, thought-provoking and, perhaps, inspirational for your own research projects as we progress toward that time, as referred to by Dr. Feynman, when the bottom starts to fill up!

\section{Reference}

Feynman RP. There's plenty of room at the bottom. Eng Sci. 1960;23(5):22-36 